\title{
An operational HF system for single site localization
}

\author{
F. Marie *, Y. Erhel ${ }^{\dagger}$ and C. Danion ${ }^{\dagger}$ \\ ${ }^{*}$ IETR University of Rennes $1,{ }^{\dagger}$ CREC St Cyr, ${ }^{\dagger}$ Company Coris, FRANCE
}

Keywords: SSL, Direction finding, MUSIC, Prime model.

\begin{abstract}
This paper presents an operational HF (3-30MHz) system designed for single site localization (SSL). An original version of the MUSIC algorithm jointly estimates the angles of arrival (azimuth and elevation) of incident waves and their polarization.

The corresponding measurements are the input data of a ray tracing software based on the PRIME model of the ionosphere which computes the estimated position of the transmitter.

Numerous radio links have been tested for long distances up to $2000 \mathrm{~km}$. A very good agreement is observed between the actual and the estimated positions of the transmitters with a standard localization error of less than $10 \%$ of the distance to the receiving system.
\end{abstract}

\section{Introduction}

Generally, radio direction finding operates on homogeneous antenna arrays, the geometrical inter element phases being directly related to the angles of arrival (AOA). In order to improve the angular resolution in the context of trans-horizon links refracted by the ionosphere, we proposed a structure of so-called heterogeneous arrays [1]. An original version of the MUSIC algorithm has been derived which estimates the incident polarizations jointly to the AOA.

The estimated AOA are considered to be the input data of a ray tracing software based on the PRIME model of the ionosphere [2]. For each incident wave detected, a possible region for the end of the corresponding path is identified on the Earth's surface. The intersection of these areas gives the estimated position of the transmitter.

Numerous experiments are carried out with transmitters located more than $1000 \mathrm{~km}$ from the receiving site. The results show a standard localization error typically equal to a few per cent of the range of the radio link under test.

\section{Heterogeneous array}

A circular uniform array of 8 active whip antennas is set up with a $20 \mathrm{~m}$ radius as shown in Fig. 1. A heterogeneous structure is obtained with the alternation of vertical and horizontal antennas, the pointing azimuth of which is different from one position to another, in order to optimize the diversity of the antennas' responses.

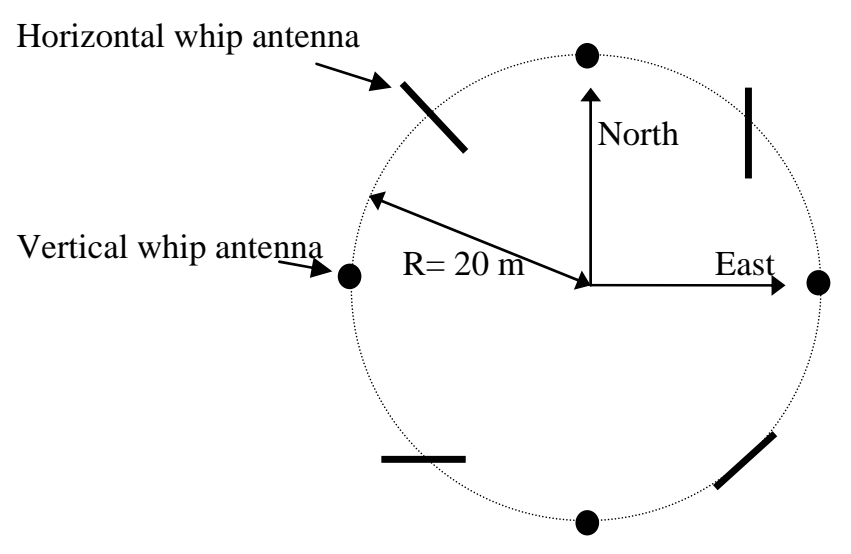

Top view

Figure 1: Heterogeneous circular array

\section{Signal processing}

After electronic calibration, the MUSIC algorithm [3] runs with base band acquisitions. In this version, the complex spatial responses of the antennas are integrated in the steering-vector projected in the noise subspace [1]. Since two polarizations (Ordinary and eXtraordinary) are expected at the exit of the ionosphere, two different pseudo-spectrums $\mathrm{PSSP}_{\mathrm{O}}$ and $\mathrm{PSSP}_{\mathrm{X}}$ are computed, demonstrating in that way the polarization sensitivity of the heterogeneous array.

\section{Angular estimations}

In order to provide reliable angular estimations, a large acquisition file (typically 120000 samples at a rate of $40 \mathrm{ksamples} / \mathrm{s}$ ) is arranged in consecutive frames for which an independent computation of the MUSIC algorithm is processed.

A set of criterions [4] checks the coherence of the successive pseudo-spectrums and rejects the artefacts, assuming that a single transmitter is operating within the bandwidth $(3 \mathrm{kHz})$ of the receivers.

One example is the constraint that the estimated azimuths for the two polarizations $\mathrm{O}$ and $\mathrm{X}$ have to be contained in a limited interval. 
The final direction finding results are the number of signals detected (resulting from a multipath propagation) and, for each of them, the mean value and the standard deviation of the estimated AOA.

\section{Single site Localization}

The PRIME model of the ionosphere (Prediction and Regional Ionospheric Modelling over Europe) has been selected for this project. It is the result of the COST 238 program (European Cooperation in the field of Scientific and Technical Research) between 1991 and 1994. This model describes the behaviour of ionospheric plasma in the mid latitudes and particularly those above Europe. From ionospheric parameters predicted by the model, the profile of vertical ionization is calculated, using Epstein functions (Fig. 2).

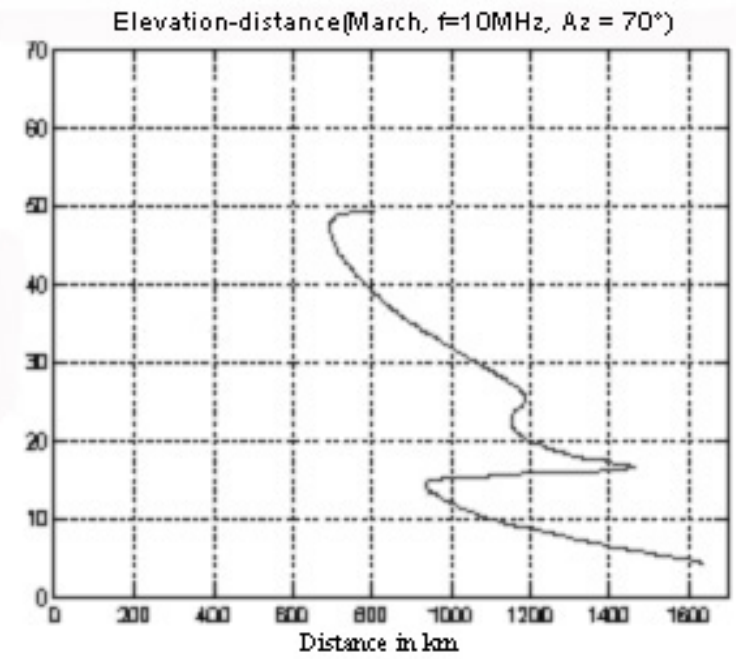

Figure 2: Vertical Ionospheric profile is made with Epstein function

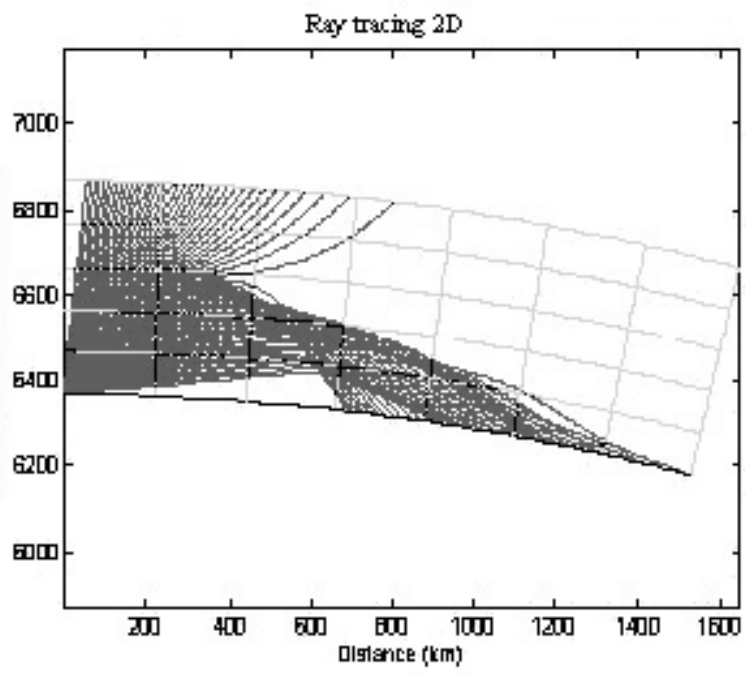

Figure 3: ray tracing
For each detected path, a ray tracing software (Fig. 3) considers the directions of arrival in a volume defined by the mean values of the AOA and their standard deviations; the corresponding ray ends define a region of the Earth's surface. When the calculation for the different incident waves is repeated, the intersection of the corresponding areas gives the possible location of the transmitter.

\section{Experimental results}

The direction finding system is set up near Orléans (France). The tests carried out localizations of H.F. transmitters located in Europe and North Africa at a maximum distance of 2000 $\mathrm{km}$. More than 100 experiments, involving 35 military beacons or broadcast transmitters, were conducted during a period of 3 weeks, at various times in the day (and during the night) and for different expected azimuths. The following figures show an example of the experiment results related to a transmitter with a carrier frequency of $9.525 \mathrm{MHz}$ and located in Warsawska (Poland).

See at the end of document:

Fig. 4 plots a couple of pseudo-spectrums (one for each polarization $\mathrm{O}$ or $\mathrm{X}$ ) picked up in the collection calculated with a long term acquisition of 3 seconds : 2 incident signals are detected with estimated azimuths both being equal to $65^{\circ}$.

Fig. 5 represents the localization chart resulting from the ray tracing. The estimated location of the transmitter is inside the red surface whereas the actual site is plotted with a black point and appears close to the estimation: this point is separated from the centre of the surface by $75 \mathrm{~km}$ when the transmitter-receiver distance equals $1410 \mathrm{~km}$.

Fig. 6 and 7 represent the couple of pseudo-spectrum and the localization chart for an Italian beacon.

More examples will be seeing during the oral presentation.

\section{Conclusion}

This article presents a global SSL project for trans-horizon communications revealing performances quite compatible with military objectives since the relative localization error appears moderate; however, the parts of it attributed either to the angular estimation or to the ionospheric model are not easy to identify.

The main originality of the direction finding system is the heterogeneous structure of the antennas array: its polarization-sensitivity reinforces the high resolution capacity of the algorithm and provides reliable estimations of the AOA in a context of multiple incident waves with a reduced angular separation. 


\section{Acknowledgements}

This study proceeded over 3 year's duration. The project's partners were company CORIS with the French Defence (DGA), the school research center of Coetquidan and the University of Rennes 1 . We thank all the partners for the success of this project. We particularly thank the DGA for its financial support.

\section{References}

[1] D. Lemur, F. Marie, L. Bertel, and Y. Erhel, 'H.F. radio direction finding operating on a heterogeneous array: principles and experimental validation', RadioScience, Jan-Feb 2004, vol 39, n¹, pp 1003-1; 100314.

[2] P.A. Bradley, 'review of COST 238 (PRIME) achievements', IEE Conference Digest 1995/204, pp 7/1-7/6 (1995).

[3] R.O. Schmidt, 'Multiple Emitter Location and Signal parameter Estimation', IEEE Transactions on Antennas and Propagation Vol. AP-34 n³, March 1986.

[4] F. Marie, L. Bertel and Y. Erhel, 'Statistical analysis of H.F. direction finding estimations' 2002 URSI General Assembly, Maastricht (N.L.), August 13-16, 2002.

\section{Authors' affiliations:}

F. MARIE : Institut d'Electronique et Télécommunications de Rennes (I.E.T.R.) Université de Rennes 1 Campus de Beaulieu 35042 RENNES France francois.marie@univrennes1.fr

Y. ERHEL : Centre de Recherches des Ecoles de St Cyr Coetquidan 56381 GUER France

yvon.erhel@st-cyr.terre.defense.gouv.fr

C. DANION : Société Coris, 2 avenue Descartes 92350 LE PLESSIS ROBINSON France

christian.danion@coris-telecom.com 


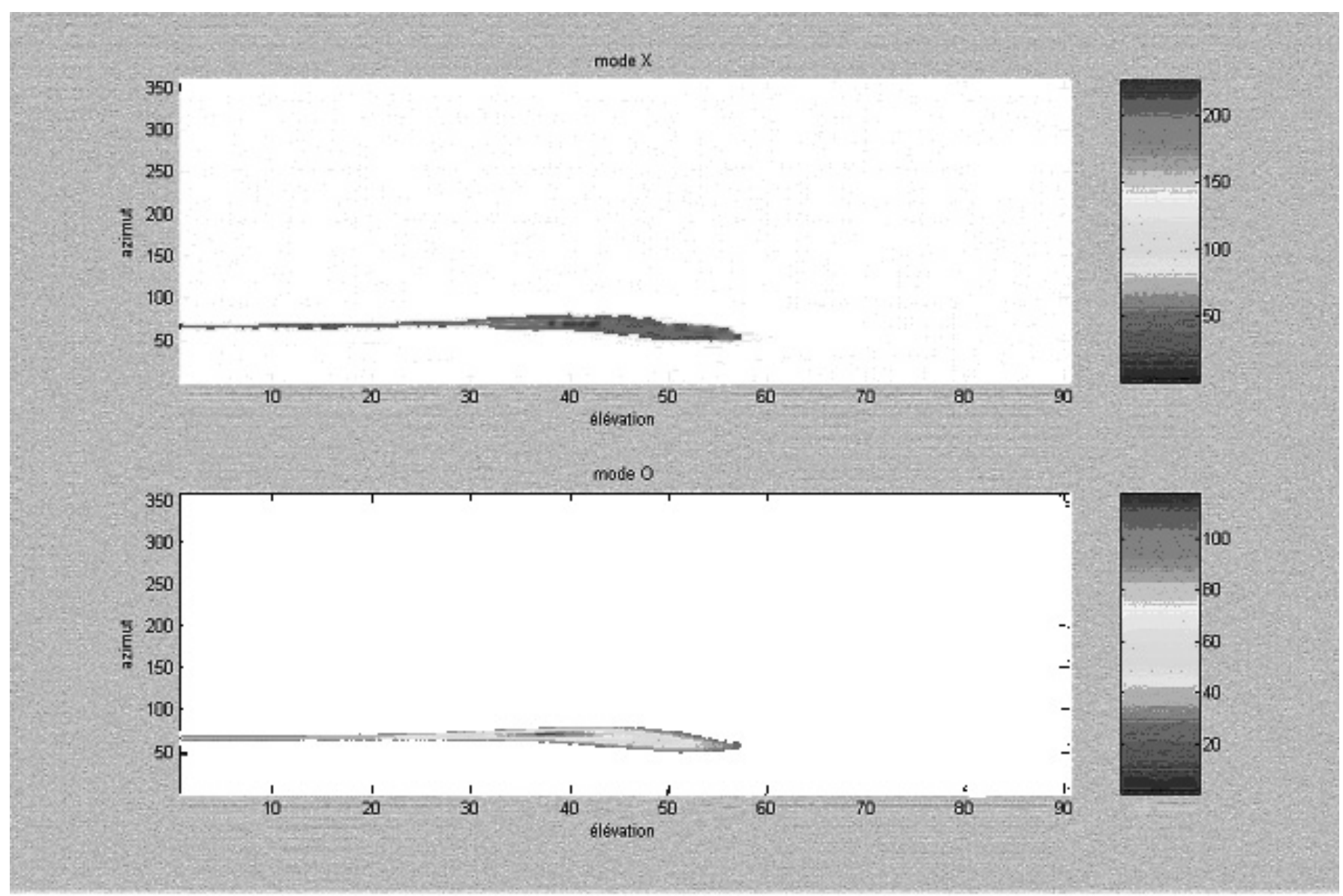

Figure 4 : Pseudo spectrums $\mathrm{O}$ and X (Warsawska acquisition)

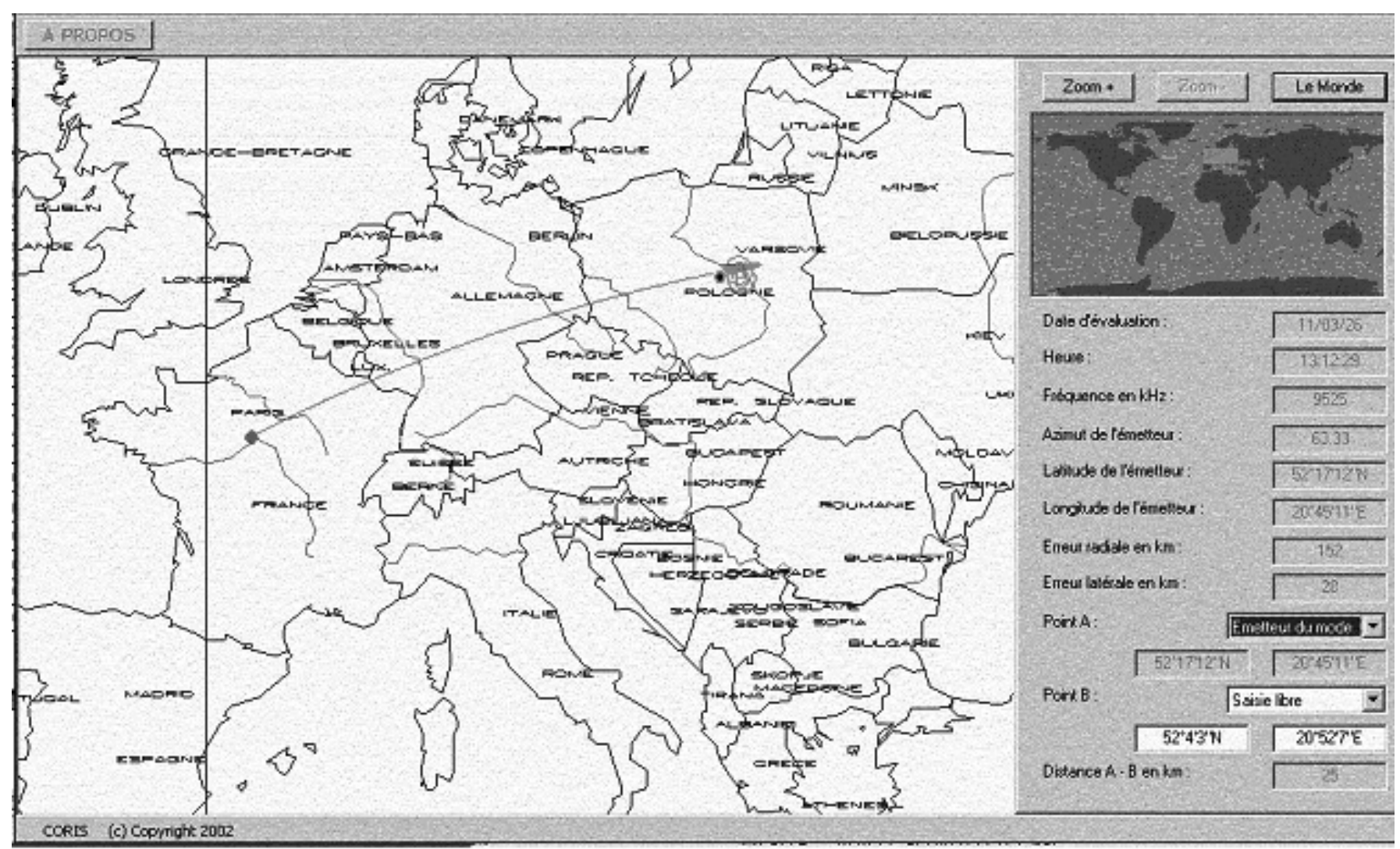

Figure 5: Estimated location of the transmitter 


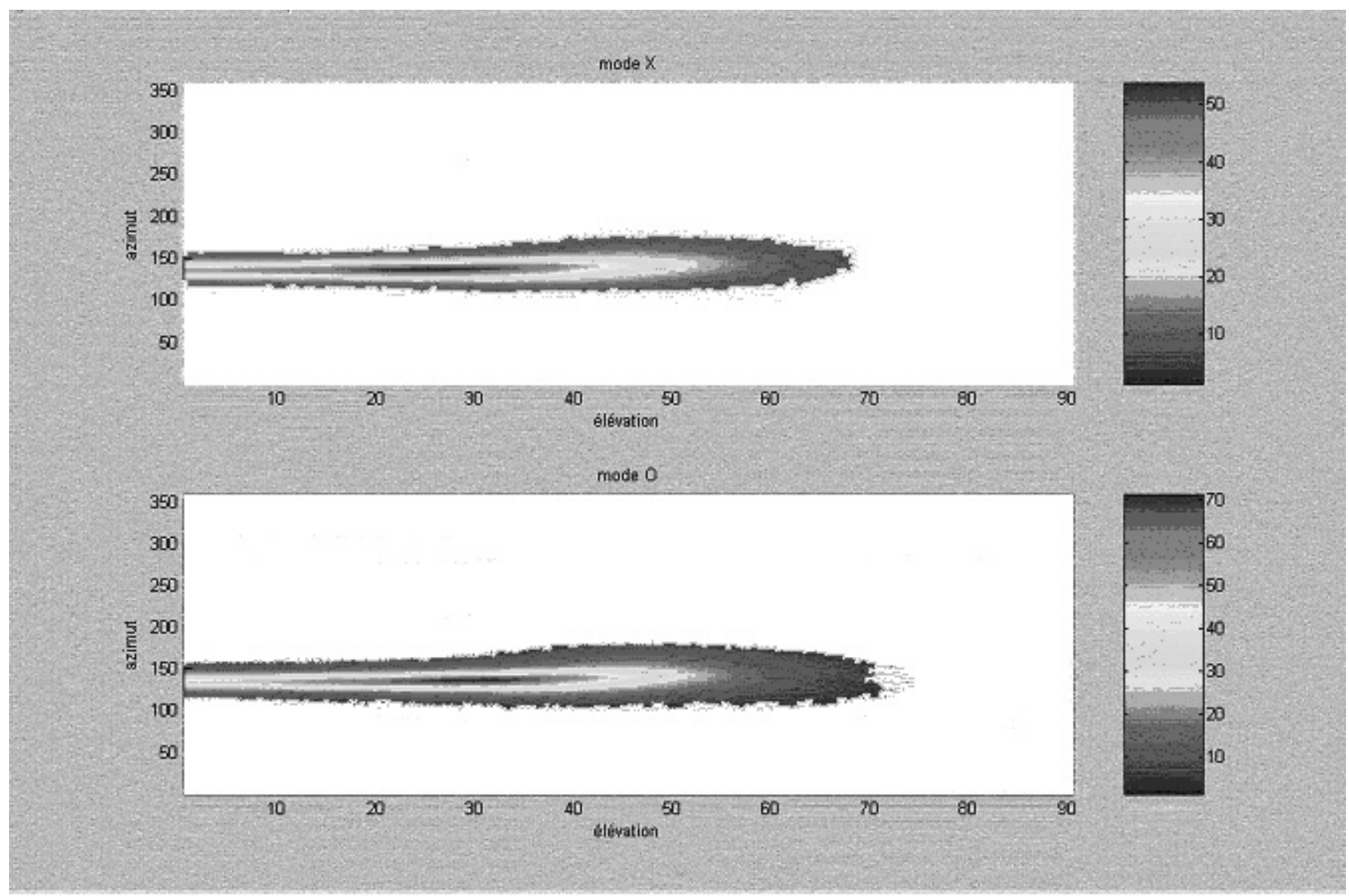

Figure 6: Pseudo spectrums $\mathrm{O}$ and $\mathrm{X}$ (Italian beacon acquisition)

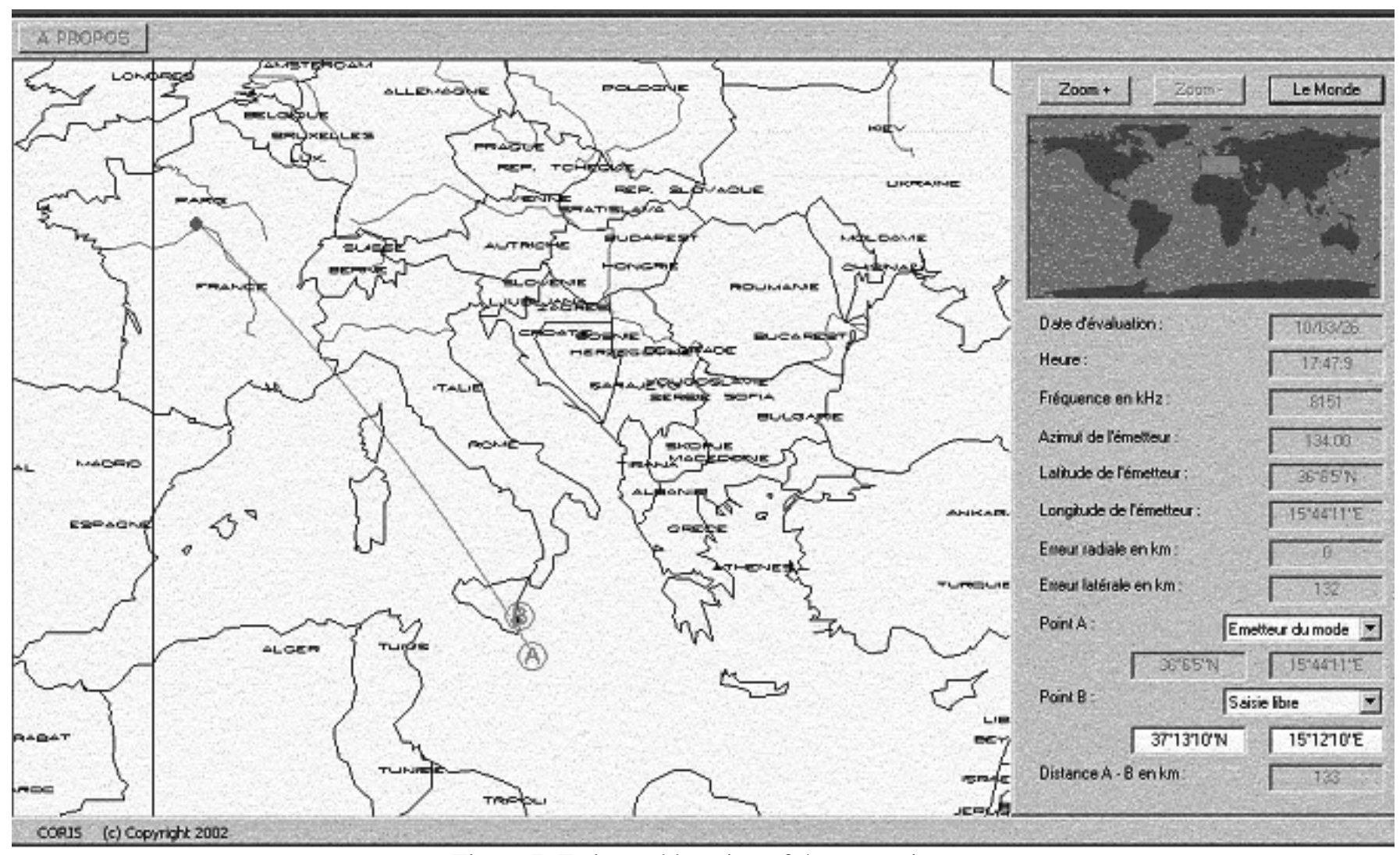

Figure 7: Estimated location of the transmitter 